\title{
Primer informe de Lutzomyia yuilli Young \& Porter, 1972 y Lutzomyia triramula (Fairchild \& Hertig 1952) (Diptera: Psychodidae) en el departamento de Caldas, Colombia
}

\author{
Daniela Vergara ${ }^{1,4}$, Lina María Carrillo ${ }^{1,2}$, Eduar Elías Bejarano ${ }^{3}$ \& Iván Darío Vélez ${ }^{1}$ \\ ${ }^{1}$ Programa de Estudio y Control de Enfermedades Tropicales - PECET, \\ Universidad de Antioquia - UDEA, \\ Calle 62, $n^{\circ}$ 52-59, A.A. 1226, Medellín, Antioquia, Colombia \\ ${ }^{2}$ Facultad de Ciencias Agrarias, Universidad de Antioquia - UDEA, \\ Carrera 75 No. 65-87, A.A. 1226, Medellín, Antioquia, Colombia \\ ${ }^{3}$ Grupo de Investigaciones Biomédicas, Universidad de Sucre - UNISUCRE, \\ Cra. 14, $n^{\circ}$ 16B-32, A.A. 406, Sincelejo, Sucre, Colombia \\ ${ }^{4}$ Autor para correspondencia: Daniela Vergara, \\ e-mail:verdaniela@gmail.com,http://medicina.udea.edu.co/pecet
}

VERGARA, D., CARRILLO, L.M., BEJARANO, E.E. \& VÉLEZ, I.D. 2008. First record of Lutzomyia yuilli Young \& Porter, 1972 and Lutzomyia triramula (Fairchild \& Hertig 1952) (Diptera: Psychodidae) in Caldas departament, Colombia. Biota Neotrop. 8(3): http://www.biotaneotropica.org.br/v8n3/en/abstract?shortcommunication+bn00708032008.

\begin{abstract}
Nine species of Lutzomyia were collected during the research of various cutaneous leishmaniasis foci in the department of Caldas, Colombia: Lutzomyia gomezi (Nitzulescu, 1931), Lu. ayrozai (Barretto \& Coutinho 1940), Lu. panamensis (Shannon 1926), Lu. bifoliata Osorno, Morales, Osorno \& Hoyos, 1970, Lu. trapidoi (Fairchild \& Hertig 1952), Lu. yuilli Young \& Porter, 1972, Lu. triramula (Fairchild \& Hertig 1952), Lu. atroclavata (Knab 1913), and Lu. trinidadensis (Newstead 1922). Two of these species constitute a new record for the department, Lu.yuilli of the subgenus Nyssomyia, and Lu. triramula of the subgenus Trichopygomyia. The specimens of $L u$. yuilli were collected using Shannon and CDC light traps in the municipality of Norcasia, while the specimens of $\mathrm{Lu}$. triramula were collected using light and sticky traps in both Norcasia and Victoria municipalities. The importance of this finding, taking into account the limited distribution of Lu. triramula in the Colombian territory and the vector capacity of $L u$. yuilli is considered.
\end{abstract}

Keywords: Phlebotominae, Leishmania, Nyssomyia, Trichopygomia, new record, Colombia.

VERGARA, D., CARRILlO, L.M., BEJARANO, E.E. \& VÉLEZ, I.D. 2008. Primer informe de Lutzomyia yuilli Young \& Porter, 1972 y Lutzomyia triramula (Fairchild \& Hertig 1952) (Diptera: Psychodidae) en el departamento de Caldas, Colombia. Biota Neotrop. 8(3): http://www.biotaneotropica.org.br/v8n3/es/ abstract?short-communication+bn00708032008.

Resumen: Durante estudios de foco en varios municipios del departamento de Caldas, Colombia, donde se han presentado casos de leishmaniosis cutánea, se recolectaron nueve especies de Lutzomyia: Lutzomyia gomezi (Nitzulescu 1931), Lu. ayrozai (Barreto \& Coutinho 1940), Lu. panamensis (Shannon 1926), Lu. bifoliata Osorno, Morales, Osorno \& Hoyos, 1970, Lu. trapidoi (Farchild \& Hertig 1952), Lu. yuilli Young \& Porter, 1952, Lu. triramula (Fairchild \& Hertig 1952), Lu. atroclavata (Knab 1913) y Lu. trinidadensis (Newstead 1922). Dos de estos taxones constituyen nuevos registros para el departamento, Lu. yuilli del subgénero Nyssomyia, y Lu. triramula del subgénero Trichopygomyia. Los individuos de Lu. yuilli se obtuvieron mediante el uso de trampas de luz tipo Shannon y CDC en el municipio de Norcasia, mientras que los de Lu. triramula se recolectaron usando trampas de luz y adhesivas en los municipios de Norcasia y Victoria. Se discute la importancia de este hallazgo teniendo en cuenta la limitada distribución de Lu. triramula en el territorio colombiano y la capacidad vectorial de $L u$. yuilli.

Palabras-clave: Phlebotominae, Leishmania, Nyssomyia, Trichopygomia, nuevo registro, Colombia. 


\section{Introducción}

La leishmaniosis es una enfermedad zoonótica causada por parásitos del género Leishmania con un ciclo de vida complejo en donde participa un huésped vertebrado, un reservorio que casi siempre es un mamífero, y un insecto vector que en América es del género Lutzomyia (Schmidt \& Roberts 2000).

Los insectos del género Lutzomyia pertenecen a la familia Psychodidae y a la subfamilia Phlebotominae. Algunos de los miembros de esta subfamilia son los responsables de la transmisión de otras enfermedades además de la leishmaniosis, como la bartonelosis y algunos arbovirus. Cerca de 460 especies de Lutzomyia han sido registradas en América y aproximadamente 30 de éstas se encuentran asociadas con la transmisión de leishmaniosis (Young \& Duncan 1994). En Colombia, se han registrado 141 especies de Lutzomyia (Bejarano 2006, Bejarano et al. 2006) desde los 0 a los $2400 \mathrm{msnm}$ en una gran variedad de ecosistemas (Montoya-Lerma \& Ferro 1999). Solo nueve de estas especies de flebotomíneos están considerados como vectores de Leishmania spp.: Lu. longipalpis (Lutz \& Neiva 1912), Lu. evansi (Nuñez-Tovar, 1924), Lu. spinicrassa Morales, Osorno, Osorno \& Hoyos, 1969, Lu. hartmanni (Fairchild \& Hertig 1957), Lu. umbratilis Ward \& Frahia, 1977, Lu. trapidoi (Fairchild \& Hertig 1952), Lu. yuilli Young \& Porter, 1972, Lu. panamensis (Shannon, 1926) y Lu. gomezi (Nitzulescu, 1931) (Santamaría et al. 2006, Montoya-Lerma \& Ferro 1999).

En Colombia la leishmaniosis es endemica y afecta principalmente a las poblaciones humanas que habitan las areas rurales y semi-urbanas del país. La transmisión ocurre generalmente en áreas boscosas donde los humanos se infectan al penetrar en los biotopos del vector. Sin embargo, se encuentran insectos vectores dentro de las viviendas, en cuyo caso la enfermedad involucra a familias enteras en las que predominan la pobreza y las dificultades para acceder a los servicios de salud, lo que genera un alto subregistro de la enfermedad (Vélez et al. 1991). Pese a ello se registraron oficialmente 8.447 casos de leishmaniosis en el año 2006 (Sivigila 2006).

Teniendo en cuenta que esta enfermedad constituye un problema de salud pública en Colombia, se hace necesario y prioritario registrar las especies de Lutzomyia presentes en las diferentes regiones del país para facilitar el control vectorial de la leishmaniosis (Bejarano et al. 2003). El presente artículo tiene como objetivo informar por primera vez el hallazgo de dos especies, Lu. yuilli y Lu. triramula (Fairchild \& Hertig 1952) en el departamento de Caldas.

\section{Materiales y Métodos}

La recolección de los individuos se hizo durante el mes de noviembre de 2006 en varios municipios del departamento de Caldas, Colombia, uno de los cuales fue Norcasia. Este municipio se encuentra ubicado a $5^{\circ} 34^{\prime} \mathrm{N}$ y a $74^{\circ} 51^{\prime} \mathrm{O}$ a una altura de $335 \mathrm{msnm}$, con una temperatura promedio de $25^{\circ} \mathrm{C}$ y una pluviosidad de $4340 \mathrm{~mm}$ anuales.

Otro de los municipios muestreados fue La Victoria. La recolección de insectos se hizo en las afueras del municipio, ubicado a $5^{\circ} 33^{\prime} \mathrm{N}$ y a $74^{\circ} 53^{\prime} \mathrm{O}$, a una altura de $550 \mathrm{msnm}$. La temperatura media de La Victoria es de $25{ }^{\circ} \mathrm{C}$, con una precipitación promedio anual de $3.717 \mathrm{~mm}$. El territorio que comprende este municipio se caracteriza por tener extensas áreas planas con temperaturas altas y su superficie corresponde a un piso térmico cálido.

En el municipio de Norcasia el muestreo se realizó usando trampas de luz tipo Shannon y CDC, mientras que en La Victoria se utilizaron trampas de papel saturado en aceite de ricino. Para las trampas Shannon se emplearon tres colectores equipados con aspiradores bucales. Las trampas CDC fueron ubicadas en el peridomicilio entre las $18 \mathrm{y}$ las 06 horas, en tanto que las trampas de papel permanecieron en el extradomicilio durante ocho días. Estas últimas se pusieron en un transecto de 186 km, a lo largo del río La Miel, desde San Miguel, en el municipio de Puerto Triunfo, Antioquia, hasta Marquetalia, en el departamento de Caldas.

Los flebotomíneos recogidos se transportaron en frascos de $1.5 \mathrm{~mL}$ con alcohol al 90\% hasta el Laboratorio de Entomología del Programa de Estudio y Control de Enfermedades Tropicales PECET de la Universidad de Antioquia. Posteriormente, los insectos fueron aclarados con una solución de ácido láctico y ácido fénico en proporción 1:1 para su posterior montaje con el medio de Hoyer en láminas portaobjeto. Para la identificación de especie se usó la clave de Young \& Duncan (1994).

\section{Resultados y Discusión}

Durante la recolecta realizada durante cuatro días con los métodos de captura CDC y Shannon, se obtuvieron 55 hembras y 5 machos de siete especies de Lutzomyia. La distribución por especies de las hembras fue la siguiente: 2 pertenecientes a Lu. gomezi, 37 a Lu. ayrozai (Barretto \& Coutinho 1940), 5 a Lu. panamensis, 4 a Lu. bifoliata, 3 a Lu. trapidoi, 3 a Lu. yuilli y 1 a Lu. triramula. Así mismo, se recolectó 1 macho de Lu. gomezi, 3 de Lu. ayrozai y 1 de Lu. bifoliata, Osorno, Morales, Osorno \& Hoyos, 1970.

Los once especímenes recogidos con las trampas de papel se distribuyeron de la siguiente manera: cuatro hembras de las especies Lu. atroclavata, Lu. gomezi, Lu. trinidadensis (Newstead 1922), y Lu. triramula, y siete machos de la especie Lu. trinidadensis. Entre las especies encontradas se destacan por su importancia vectorial para la leishmaniosis, Lu. gomezi, Lu. panamensis, Lu. trapidoi y Lu. yuilli. Esta última no había sido registrada antes para el departamento de Caldas

Lu. yuilli pertenece al subgénero Nyssomyia de reconocida importancia médica debido a que la mayoría de sus especies son antropofílicas y vectores de algunos arbovirus, así como de leishmaniosis. Esta especie tiene una amplia distribución en varios países de América Latina como Colombia, Perú, Ecuador, Brasil, Bolivia y Venezuela (Young \& Duncan 1994).

En Colombia, Lu. yuilli se ha encontrado tanto en los bosques, como en el peri e intradomicilio (Cortés 2006, Sandoval et al. 2006, Santamaría et al. 2006, Barreto et al. 2000). Uno de los hallazgos más importantes de Lu. yuilli es el aislamiento de Leishmania (Viannia) panamensis en individuos capturados en el intra y peri domicilio del departamento de Boyacá (Santamaría et al. 2006, Young \& Duncan 1994). Esto indica que $\mathrm{Lu}$. yuilli probablemente participa activamente en la transmisión de esta especie de Leishmania, que causa mas del $50 \%$ de los casos de leishmaniosis cutánea en el país (Vélez et al. datos sin publicar).

Otra de las especies encontradas y no reportada antes en el departamento de Caldas es Lu. triramula, mientras que Lu. ayrozai es registrada por primera vez en el municipio de Norcasia (Barreto et al. 2006). Esta última pertenece al subgénero Psychodopygus (Mangabeira 1941), de amplia distribución en América, y se encuentra en Panamá, Colombia, Venezuela, Trinidad, Guyana Francesa, Ecuador, Perú, Brasil y Bolivia. Se ha comprobado su importancia epidemiológica en la Amazonia brasileña donde es muy antropofílica y ha sido incriminada como vector de Le (V.) naiffi (Afonso et al. 2007).

Por último, Lu. triramula se encuentra agrupada en el subgénero Trichopygomyia. Este subgénero se caracteriza por tener individuos que se ven atraídos por la luz (Young \& Duncan 1994) y se han encontrado en cuevas de armadillos (Christensen \& Vasquez 1982). Aunque la importancia médica del subgénero es desconocida debido a que las hembras no son antropofílicas, Hashiguchi et al. (1992) capturaron a miembros de Trichopygomyia utilizando cebo humano. 
Esta especie se encuentra distribuida en América desde Guatemala hasta Ecuador.

Desde el punto de vista ecológico, es importante notar que la mayoría de los registros de Lu. yuilli y Lu. triramula en Colombia (Bejarano 2006), provienen de ecosistemas de bosque húmedo, lo que coincide en parte con lo encontrado en el presente estudio. Excepcionalmente, Lu. yuilli también ha sido registrada en zonas de bosque seco tropical del norte del país (Cortés 2006).

El hallazgo de Lu. yuilli y Lu. triramula en el departamento de Caldas aumenta el número de especies reportadas de Lutzomyia en este departamento a 27. Lu. yuilli se ha registrado en siete departamentos de Colombia, aunque es probable que su ámbito geográfico sea mayor, por lo cual es necesario continuar su estudio con el fin de establecer su importancia médica en otras regiones del país. El nuevo registro de $\mathrm{Lu}$. triramula eleva el número de departamentos donde se ha informado su presencia a siete. Estos hallazgos contribuyen a la actualización de la distribución de las especies de flebotomíneos en el país, conocimiento fundamental para el entendimiento de su ecología y el posterior control de los mismos.

\section{Agradecimientos}

Este trabajo se realizó dentro del marco del convenio realizado entre Isagen y el Programa de Estudio y Control de Enfermedades Tropicales (PECET) de la Universidad de Antioquia. Agradecemos a todo el personal de Isagen en la Hidroelectrica Miel, a la Doctora Martha Wolff por su ayuda y a las comunidades de Norcasia, Victoria y demás municipios por su colaboración.

\section{Referencias Bibliográficas}

AFONSO, M.M.S., WAGNER, A.C., AZEVEDO, A.C.R., DA COSTA, S.M., MAURÍCIO, L., VILELA, M.L. \& RANGEL, E.F. 2007. Data on sand fly fauna (Diptera, Psychodidae, Phlebotominae) in Itatiaia National Park, Rio de Janeiro State, Brazil. Cad. Saúde Pública 23(3):725-730.

BARRETO, M., BURBANO, M.E. \& BARRETO, P. 2000. Lutzomyia Sand Flies (Diptera: Psychodidae) from Middle and Lower Putumayo Department, Colombia, with New Records to the Country. Mem. Inst. Oswaldo Cruz 95(5):633-639.

BARRETO, M., BURBANO, M.E. \& BARRETO, P. 2006. Registros de Lutzomyia (Diptera: Psychodidae) en nuevas localidades de Colombia. Colomb. Med. 37(1):39-45.
BEJARANO, E.E. 2006. Lista actualizada de los psicódidos (Diptera: Psychodidae) de Colombia. Folia Entomol. Mex. 45(1):47-56.

BEJARANO, E.E., DUQUE, P. \& VÉLEZ, I.D. 2006. Redescripción de la hembra de Lutzomyia vattierae (Diptera: Psychodidae, Phlebotominae) de la serranía de La Macarena, Colombia. Biomédica 26(4):556-561.

CHRISTENSEN, H.A. \& VASQUES, A.M. 1982. The tree-buttress biotope: a pathobiocenose of Leishmania braziliensis. Am. J. Trop. Med. Hyg. 31(2):243-251

CORTÉS, L.A. 2006. Foco de leishmaniasis en El Hobo, municipio de El Carmen de Bolívar, Bolívar, Colombia. Biomédica 26(Supl.1):236-241.

HASHIGUCHI, Y., CHILLER, T., INCHAUSTI, A., DE ARIAS, A., KAWABATA, M., ALEXANDER, J.B. 1992. Phlebotomine sandfly species in Paraguay and their infection with Leishmania. Am. J. Trop. Med. Hyg. 86(2):175-180.

SANDOVAL, C.M., GUTIÉRREZ, R., CÁRDENAS, R., FERRO, C. 2006 Especies del género Lutzomyia (Psychodidae, Phlebotominae) en áreas de transmisión de leishmaniasis tegumentaria visceral en el departamento de Santander, en la cordillera oriental de los Andes colombianos. Biomédica 26(Supl.1):218-227.

SANTAMARÍA, E., PONCE, N., ZIPA, Y., FERRO, C. 2006 Presencia en el peridomicilio de vectores infectados con Leishmania (Viannia) panamensis en dos focos endémicos en el occidente de Boyacá, piedemonte del valle del Magdalena medio, Colombia. Biomédica 26(Supl.1):82-94.

SCHMIDT, G.D., ROBERTS, L.S. 2000. Foundations of Parasitology. McGraw - Hill Higher Education, Boston, p.70-78.

SIVIGILA. Instituto Nacional de Salud, Subdirección de Vigilancia y Control en Salud Pública. 2006. Sistema de Vigilancia en Salud Pública Sivigila. Semana epidemiológica. Santafé de Bogotá, 52p.

VÉLEZ, I.D., WOLFF, M., VALDERRAMA, R., ESCOBAR, J.P., OSORIO, L. 1991 Community and environmental risk factors associated with cutaneus leishmaniosis in Montebello, Antioquia, Colombia. In Leishmaniasis Control Strategies. IDR, Mexico. p.261-74.

YOUNG, D.G. \& DUNCAN, M.A. 1994. Guide to the identification and geographic distribution of Lutzomyia sandflies in Mexico, the West Indies, Central and South America (Diptera: Psychodidae). Associated Publishers American Entomological Institute, Gainesville, 881p.

Recibido en 14/11/07

Versión revisada en 20/06/08

Publicado en 10/07/08 